\title{
Therapeutic temperature management (TTM): post-resuscitation care for adult cardiac arrest, with recommendations from the National TTM Workgroup
}

Siew Hon Benjamin Leong ${ }^{1}$, MBBS, MRCSEd, Enoch $\underline{\mathrm{Chan}^{2}}$, BSc(N), MN, Benjamin Choon Heng $\underline{\mathrm{Ho}}^{3}$, MBBS, MRCP, Colin $\underline{Y e O}^{4}$, MBBS, MRCP, Sennen Lew $\underline{L}^{5}$, MBBS, MRCP, Duu Wen Sewa ${ }^{6}$, MBBS, MRCP, Shir Lynn $\underline{\operatorname{Lim}}^{6}$, MBBS, MMed, Chee Wan $\underline{L e e}^{7}$, MBBS(Hons), MRCP, Pow Li Chia ${ }^{8}$, MBBS, MRCP, Tien Siang Eric $\underline{\operatorname{Lim}}^{9}$, MB Bhir, MRCP, Eng Kiang Lee $\underline{10}^{10}$, MBBS, FANZCA, Marcus Eng Hock $\underline{\text { Ong }}^{11,12}$, MBBS, MPH

\begin{abstract}
Therapeutic temperature management (TTM) was strongly recommended by the 2015 International Liaison Committee on Resuscitation as a component of post-resuscitation care. It has been known to be effective in improving the survival rate and neurologic functional outcome of patients after cardiac arrest. In an effort to increase local adoption of TTM as a standard of post-resuscitation care, this paper discusses and makes recommendations on the treatment for local providers.
\end{abstract}

Keywords: cardiac arrest, post-resuscitation, therapeutic temperature management

\section{INTRODUCTION}

There has been increasing awareness in the community of the need to improve out-of-hospital cardiac arrest (OHCA) and in-hospital cardiac arrest (IHCA) survival in Singapore. Numerous factors can impact survival and these may be organised according to the chain of survival concept, which includes early access, early cardiopulmonary resuscitation (CPR), early defibrillation, early advanced care and post-resuscitation care.(1) Despite CPR and defibrillation, a large proportion of the victims who survive to hospital admission still die.

Therapeutic hypothermia or therapeutic temperature management (TTM) and its potential benefits have been known for a few decades, but only gained wide support after two landmark studies were published in 2002. These studies showed significant improvement in both survival and neurological outcomes for post-cardiac arrest patients. ${ }^{(2,3)}$ By cooling the patient's body after resuscitation, reperfusion injury due to inflammation and free radical production is suppressed, thereby reducing cell death and injury to the brain and vital organs. ${ }^{(4)}$ The use of TTM is strongly recommended in the 2015 update by the International Liaison Committee on Resuscitation for patients with ventricular fibrillation (VF) or pulseless ventricular tachycardia $(\mathrm{pVT}){ }^{(5)}$

Although TTM is a recommended standard of care, its adoption in Singapore for cardiac arrest patients is not widespread. The local awareness and understanding of its science and evidence, as well as experience in its implementation, remain limited.

\section{PATHOPHYSIOLOGY AND EFFECTS OF TTM}

After resuscitation from cardiac arrest, a complex series of processes occurs within minutes to hours, leading to cellular level injury. Cerebral ischaemic damage happens within seconds when cerebral circulation is compromised. Glucose and adenosine triphosphate are depleted, and cells begin to lose structural integrity, leading to mitochondrial damage and loss of calcium homeostasis. Cerebral reperfusion injury occurs when the restoration of oxygenation leads to the accumulation of oxygen free radicals and activation of degradative enzymes. . $^{(3,4,6)}$

Therapeutic hypothermia reduces the release of excitatory amino acids, free radicals and the intracellular consequences of excitotoxin exposure. ${ }^{(6)}$ It also decreases the cerebral metabolic rate by $6 \%$ for every $1{ }^{\circ} \mathrm{C}$ drop in core body temperature, thus reducing cerebral oxygen demand.(7)

\section{RECOMMENDATIONS}

\section{Indications for TTM}

TTM is recommended for all patients with return of spontaneous circulation (ROSC) from cardiac arrest who have abnormal conscious levels during the immediate post-arrest period.

Cooling to mild hypothermia temperatures has been shown to have survival and neurological benefits for cardiac arrest patients with shockable rhythms. Two randomised controlled trials (RCTs) provided clear evidence that TTM should be used for patients with shockable rhythms. ${ }^{(2,3)} \mathrm{A}$ decrease in mortality and improvement in neurological outcome were shown in patients presenting with ventricular tachycardia (VT) and pVT rhythms, who remained comatose after ROSC and received TTM. ${ }^{(2,3)}$ In these studies, $49 \%-55 \%$ of patients who received some form of TTM survived with favourable neurological outcome, compared to the $26 \%-39 \%$ of patients who did not receive TTM.

\footnotetext{
${ }^{1}$ Emergency Medicine Department, National University Health System, ${ }^{2}$ Unit for Prehospital Emergency Care, Singapore General Hospital, ${ }^{3}$ Medical Intensive Care Unit, Tan Tock Seng Hospital, ${ }^{4}$ Cardiology Department, Changi General Hospital, ${ }^{5}$ Medical Intensive Care Unit, Khoo Teck Puat Hospital, ${ }^{6}$ Medical Intensive Care Unit, Singapore General Hospital, ${ }^{7}$ Cardiology Department, Khoo Teck Puat Hospital, ${ }^{8}$ Cardiology Department, Tan Tock Seng Hospital, ${ }^{9}$ Cardiology Department, National Heart Centre Singapore, ${ }^{10}$ Medical Intensive Care Unit, Ng Teng Fong General Hospital, ${ }^{11}$ Department of Emergency Medicine, Singapore General Hospital, ${ }^{12} \mathrm{Health}$ Services and Systems Research, Duke-NUS Medical School, Singapore

Correspondence: Dr Benjamin Leong, Senior Consultant, Emergency Medicine Department, National University Health System, 5 Lower Kent Ridge Road, Singapore 119074. Benjamin_SH_LEONG@nuhs.edu.sg
} 
For patients presenting with nonshockable rhythms or IHCA, the evidence is less consistent. ${ }^{(5,8)} \mathrm{A}$ meta-analysis by Kim et al ${ }^{(9)}$ found that TTM was beneficial for cases of nonshockable rhythms, although the included studies had substantial risk for bias. Although the level of evidence is low, TTM remains recommended for these patients.

\section{Target temperature for TTM}

A target temperature of $33^{\circ} \mathrm{C}-36^{\circ} \mathrm{C}$ should be selected and maintained within a $1^{\circ} \mathrm{C}$ variation for the duration of TTM. For patients with $\mathrm{OHCA}$ due to shockable rhythms, a lower target temperature of $33^{\circ} \mathrm{C}$ is recommended. If contraindications to lower temperatures are present, a higher target temperature of $36^{\circ} \mathrm{C}$ may be used. Fever should be prevented in all patients with cardiac arrest and abnormal consciousness levels, and thus $36^{\circ} \mathrm{C}$ should be the minimum target temperature of cooling for all patients. Early studies have attempted the use of deep hypothermia of below $30^{\circ} \mathrm{C}$ to provide neurological protection. Deep hypothermia (below $25^{\circ} \mathrm{C}-28^{\circ} \mathrm{C}$ ) was suggested purely with the assumption that hypothermia induced lower metabolism. It was later found in animal studies that mild hypothermia provided significant protective effects with much fewer side effects. ${ }^{(4)}$

Since the two RCTs in 2002, ${ }^{(2,3)}$ the target temperature of $32^{\circ} \mathrm{C}-34^{\circ} \mathrm{C}$ has been adopted as the recommended target..$^{(2,3,5,8)}$ A subsequent $\mathrm{RCT}$, which compared the target temperatures of $33^{\circ} \mathrm{C}$ vs. $36^{\circ} \mathrm{C}$ for cardiac arrests of all rhythms, found no difference in outcomes. ${ }^{(10)}$ In the Nielsen et al study, both the intervention and the control groups were given some form of TTM. The only difference was the target temperature. ${ }^{(10)}$ This was in contrast to the studies by the Hypothermia after Cardiac Arrest study group ${ }^{(2}$ and Bernard et $\mathrm{al}_{,}{ }^{(3)}$ where only the intervention groups received TTM; the control groups were reported to have temperatures averaging $38^{\circ} \mathrm{C}$. In light of the original evidence that TTM at a target temperature of $32^{\circ} \mathrm{C}-34^{\circ} \mathrm{C}$ has been proven to be superior to no TTM for shockable rhythms, a lower target temperature of $33^{\circ} \mathrm{C}$ remains recommended for this group. However, in the presence of contraindications such as sepsis, coagulopathy and cardiac dysrhythmias, a higher target of $36^{\circ} \mathrm{C}$ is acceptable. For patients with nonshockable rhythms or IHCA, a temperature target of $36^{\circ} \mathrm{C}$ is acceptable. Fever in the post-arrest period has been associated with poorer outcomes ${ }^{(11,12)}$ and should be avoided in all groups.

\section{Duration of TTM and rewarming}

TTM should be induced and maintained at the selected target temperature for 24 hours, and rewarmed gradually at a rate not faster than $0.5^{\circ} \mathrm{C}$ per hour. Every hour of delay in TTM after ROSC increases mortality by $20 \%$. Thus, TTM should be initiated as soon as possible after ROSC. While there is low-quality evidence regarding the duration of cooling, most studies have suggested that rewarming should begin 24 hours after the patient has reached the target temperature. ${ }^{(6,8)}$ Patients should not be rewarmed rapidly, as rapid rewarming has been associated with complications, including hypotension, electrolyte changes and dysrhythmias. It is recommended that rewarming should occur at a rate not faster than $0.5^{\circ} \mathrm{C}$ every hour until normothermia.

\section{COMPLICATIONS OF TTM}

\section{Cardiovascular}

There may be a decrease in cardiac output by $25 \%-40 \%$ due to a decrease in heart rate during hypothermia. The central venous pressure, arterial resistance and blood pressure may rise due to vasoconstriction of peripheral arteries and arterioles induced by hypothermia. ${ }^{(13,14)}$ Bradycardia is the most common arrhythmia when cooling begins. However, temperatures below $30^{\circ} \mathrm{C}$ are associated with increased risk of atrial fibrillation, VF and asystole. Bradycardia should be treated if it is associated with hypotension. ${ }^{(6)}$ Hypotension is common during rewarming. During cooling, cold diuresis and vasoconstriction result in a reduction of intravascular volumes. When the patient is rewarmed, vasodilation from reversal of vasoconstriction and post-resuscitation inflammatory release, as well as reduced intravascular volume, increase the risk of hypotension. Direct cardiac dysfunction from the initial ischaemic insult may also worsen hypotension. In the absence of cardiac or renal failure, fluid replacement should be initiated to mitigate hypotension, and to avoid cerebral hypotension and recurrent hypoperfusion.

\section{Shivering}

Shivering is a common reflex to cooling and typically occurs during changes in temperature of between $35^{\circ} \mathrm{C}$ and $37^{\circ} \mathrm{C}$. Once a patient achieves the target temperature of $32^{\circ} \mathrm{C}-34^{\circ} \mathrm{C}$, shivering is less common. ${ }^{(6)}$ It should be recognised and controlled early, as it increases metabolic demand and prevents or delays achievement of target temperatures. Sedation and analgesia help to reduce shivering. Paralytic agents may also be needed to suppress the shivering reflex. If used, they must be given together with sedative and analgesic agents to avoid patient awareness during the paralysis, which may be extremely distressing. Neurological monitoring during paralysis is also essential. This may include the use of continuous electroencephalogram to detect conscious level changes and nonconvulsive seizures. Warming of the peripheries (e.g. using mittens, stockings, hot packs, blankets on the hands/ feet) may also help to reduce shivering.

\section{Infections}

Hypothermia impairs neutrophil and macrophage function, and suppresses inflammatory mediators. ${ }^{(15,16)}$ Owing to the active cooling process, recognition of fever is not possible. Pneumonia and sepsis have been found to be increased during hypothermia. ${ }^{(17)}$ Therefore, care must be taken to monitor and treat infections.

\section{Endocrine effects}

Hypothermia decreases insulin secretion and increases insulin resistance, causing hyperglycaemia in patients receiving TTM. The glucose level of patients can fall precipitously during rewarming. Blood glucose should thus be monitored regularly and actively managed.

\section{Renal}

Owing to fluid and electrolyte shifts during cooling and rewarming, electrolyte levels may change significantly. Potassium 
levels may fall from diuresis or cellular shifts during cooling, and potassium may shift back into the bloodstream during rewarming. Patients with renal failure may need to be dialysed prior to rewarming. Calcium, magnesium and phosphate levels may also be affected. Therefore, close monitoring of electrolytes during TTM is crucial.

\section{Gastrointestinal}

Gastrointestinal motility is reduced during TTM and there may be an increased likelihood of stress ulcers. Liver and pancreatitic dysfunction such as raised transaminases and amylase may occur.

\section{Haematological}

Platelet counts, platelet function and other clotting factors of the patient can be affected by hypothermia, and this may induce mild bleeding diathesis. ${ }^{(14)}$ The effect will not show in standard coagulation tests unless they are performed at the patient's actual core temperature. While no clinically significant bleeding has been reported in large clinical trials, the benefit to neurological function should be assessed against the severity and location of bleeding. ${ }^{(8)}$

\section{Pharmacological}

Drug metabolism is impaired during hypothermia owing to reduced hepatic clearance, and the effects may be prolonged.

\section{Hyperthermia}

Fever is a common complication in patients with various types of neurological injury. It should be prevented for at least 72 hours after rewarming. Hyperthermia is harmful and leads to a worse neurological outcome.

\section{Peripheral complications}

Direct cold injury (depending on the cooling device used) and peripheral vasoconstriction can lead to skin necrosis, gangrene of digits/limbs and infections. Good skin care and monitoring is important.

\section{LOCAL EVIDENCE FOR TTM}

A local study showed that survival to discharge trended higher in patients who underwent TTM (TTM 44.0\% vs. control $16.0 \% ; p=0.06) .{ }^{(18)}$ Survivors in the intervention group had good neurological recovery (Cerebral Performance Category 1/2: 63.6\%; Overall Performance Category 1/2: 54.5\%). Patients who were cooled using external methods (cooling pads) compared to internal methods (cooling catheters) appeared to be more likely to have both overcooling (internal 33.3\% vs. external 50.0\%) and undercooling (internal $88.9 \%$ vs. external $93.8 \%$ ). Rebound hyperthermia (defined as temperature overshoot $\geq 38.0^{\circ} \mathrm{C}$ ) was observed in only $4(16.0 \%)$ patients, all of whom were on external cooling. ${ }^{(18)}$ TTM has also been incorporated into local protocols for extracorporeal membrane oxygenation and acute percutaneous coronary intervention.

\section{CONCLUSION}

TTM is an important component in post-resuscitation care. Evidence has shown that mild hypothermia is effective in improving the neurological function of patients who remain comatose post-ROSC. TTM is recommended for all post-ROSC patients regardless of initial shockable or nonshockable rhythm, as well as in both OHCA and IHCA.

\section{REFERENCES}

1. Neumar RW, Shuster M, Callaway CW, et al. Part 1: Executive Summary: 2015 American Heart Association Guidelines Update for Cardiopulmonary Resuscitation and Emergency Cardiovascular Care. Circulation 2015; 132(18 Suppl 2):S315-67.

2. Hypothermia after Cardiac Arrest Study Group. Mild therapeutic hypothermia to improve the neurologic outcome after cardiac arrest. N Engl J Med 2002; 346:549-56.

3. Bernard SA, Gray TW, Buist MD, et al. Treatment of comatose survivors of out-of-hospital cardiac arrest with induced hypothermia. N Engl J Med 2002; 346:557-63.

4. Polderman KH. Mechanisms of action, physiological effects, and complications of hypothermia. Crit Care Med 2009; 37(7 Suppl):S186-202.

5. Callaway CW, Donnino MW, Fink EL, et al. Part 8: Post-Cardiac Arrest Care: 2015 American Heart Association Guidelines Update for Cardiopulmonary Resuscitation and Emergency Cardiovascular Care. Circulation 2015; 132(Suppl 2):S465-82.

6. Scirica BM. Therapeutic hypothermia after cardiac arrest. Circulation 2013; 127:244-50.

7. Steen PA, Newberg L, Milde JH, Michenfelder JD. Hypothermia and barbiturates: individual and combined effects on canine cerebral oxygen consumption. Anesthesiology 1983; 58:527-32.

8. Howes D, Gray SH, Brooks SC, et al. Canadian Guidelines for the use of targeted temperature management (therapeutic hypothermia) after cardiac arrest: a joint statement from The Canadian Critical Care Society (CCCS), Canadian Neurocritical Care Society (CNCCS), and the Canadian Critical Care Trials Group (CCCTG). Resuscitation 2016; 98:48-63.

9. Kim YM, Yim HW, Jeong SH, Klem ML, Callaway CW. Does therapeutic hypothermia benefit adult cardiac arrest patients presenting with non-shockable initial rhythms?: a systematic review and meta-analysis of randomized and non-randomized studies. Resuscitation 2012; 83:188-96.

10. Nielsen N, Wetterslev J, Cronberg T, et al; TTM Trial Investigators. Targeted temperature management at $33^{\circ} \mathrm{C}$ versus $36^{\circ} \mathrm{C}$ after cardiac arrest. $\mathrm{N}$ Engl J Med 2013; 369:2197-206.

11. Zeiner A, Holzer M, Sterz F, et al. Hyperthermia after cardiac arrest is associated with an unfavorable neurologic outcome. Arch Intern Med 2001; 161:2007-12.

12. Gebhardt K, Guyette FX, Doshi AA, Callaway CW, Rittenberger JC; Post Cardiac Arrest Service. Prevalence and effect of fever on outcome following resuscitation from cardiac arrest. Resuscitation 2013; 84:1062-7.

13. Chin CT, Wong A. Controlled hypothermia in post-resuscitation management: what is so cool about it? Singapore Med J 2011; 52:603-6.

14. Polderman $\mathrm{KH}$, Herold I. Therapeutic hypothermia and controlled normothermia in the intensive care unit: practical considerations, side effects, and cooling methods. Crit Care Med 2009; 37:1101-20.

15. Polderman $\mathrm{KH}$. Application of therapeutic hypothermia in the intensive care unit Opportunities and pitfalls of a promising treatment modality--Part 2: practical aspects and side effects. Intensive Care Med 2004; 30:757-69.

16. Mongardon N, Perbet S, Lemiale $\mathrm{V}$, et al. Infectious complications in out-ofhospital cardiac arrest patients in the therapeutic hypothermia era. Crit Care Med 2011; 39:1359-64.

17. Geurts M, Macleod MR, Kollmar R, Kremer PH, van der Worp HB. Therapeutic hypothermia and the risk of infection: a systematic review and meta-analysis. Crit Care Med 2014; 42:231-42.

18. Ng M, Wong AS, Chew HC, et al. Pilot prospective study of therapeutic hypothermia for treatment of post-cardiac arrest patients. Int J Cardiol 2014; 173:612-3. 